\title{
VAC.I6 - Immunogenicity of an inactivated Yellow Fever vaccine in golden -headed lion tamarin (Leontopithecus chrysomelas)
}

André Tavares da Silva Fernandes ${ }^{1 *}$; Luciane Pinto Gaspar ${ }^{1}$; Mariana Pierre de Barros Gomes ${ }^{1}$ Marisol Simões ${ }^{1}$; José Henrique Resende Linhares ${ }^{1}$; Renato Marchevsky ${ }^{1}$; Silvia Bahadian Moreira $^{2}$; Alcides Pissinatti²; Elena Caride ${ }^{1}$; Marcos da Silva Freire ${ }^{1}$.

1Fiocruz/Bio-Manguinhos;

2Centro de Primatologia do Rio de Janeiro.

Introduction: Yellow Fever (YF) is a viral hemorrhagic disease endemic in tropical areas of South America and Africa, known to cause illness and death in humans and non-human primates (NHP). Vaccination is the best preventive measure against YF. Attenuated YF 17DD vaccine has been produced since 1937 by Bio-Manguinhos/Fiocruz, being the most effective and safest vaccine ever developed. However, some adverse events are associated with vaccination, such as viscerotropic disease, anaphylactic reactions and neurological disease. Between July 2017 and June 2018, a severe YF outbreak occurred in southeastern Brazil since 1947 resulting in 1,376 confirmed cases in humans with 483 deaths, and 864 confirmed epizooties in NHP. There is a strong demand for the development of a new vaccine for YF, as effective as the current one and safer, that is unable to cause viral disease in immunized population. Moreover, the absence of a vaccine available for NHP strengthens the need for studies to develop a vaccine for this purpose.

Objective: In the present study, the immunogenicity of an inactivated 17DD vaccine in goldenheaded lion tamarin (Leontopithecus chrysomelas) was evaluated by the determination of the neutralizing antibody titers $\left(\mathrm{PRNT}_{50}\right)$.

Methodology: The YF vaccine was inactivated with $ß$-propiolactone and adsorbed to different adjuvants. NHPs were immunized by intramuscular route with three doses of 17DD inactivated with aluminum hydroxide adjuvant or with two doses of 17DD inactivated with AddaVax ${ }^{\mathrm{TM}}$ adjuvant. NHP experiments were approved by the Animal Care and Use Committee (CEUAUNIFESO 470/2018) and environmental license was authorized by the Brazilian Ministry of the Environment (ICMBio-SISBIO 60511-2/2018).

Results: PRNT titers in serum samples from animals after the second and third immunization with 17DD inactivated with aluminum hydroxide adjuvant showed PRNT titers just above the detection limit of the assay $\left(\mathrm{PRNT}_{50}\right.$ titer $\left.>5\right)$. On the other hand, immunization with 17DD inactivated with AddaVax ${ }^{\mathrm{TM}}$ adjuvant did not elicit robust antibody titer responses, even after the second dose. The overall seroconversion rate was also considerably higher in the group that received vaccine schedule with aluminum hydroxide when compared with the group that received vaccine with AddaVax ${ }^{\mathrm{TM}}$, where $83.3 \%$ and $0.0 \%$ individuals seroconverted, respectively. As expected, the control groups immunized with different doses of the live attenuated vaccine (groups 1, 2 and 3) showed 100\%, 40\% and $83.3 \%$ of seroconversion ninety days after inoculation, respectively.

Conclusion: These data indicate the potential of this inactivated antigen for the development of a non-infectious YF vaccine. One year after the first immunization, all the groups will be challenged using attenuated $17 \mathrm{DD}$ vaccine to evaluate the protective efficacy of the inactivated vaccine.

Keywords: Yellow Fever; inactivated vaccine; preclinical study 